\title{
DISTRIBUIÇÃO VON MISES NA AVALIAÇÃO DE DADOS APÍCOLAS
}

\author{
VON MISES DISTRIBUTION IN THE EVALUATION OF APICULTURAL DATA
}

\author{
Brighenti, C.R.G. ${ }^{1 *}$; Brighenti, D.M. ${ }^{1 A} ;$ Miranda, Y.S.S. de ${ }^{1 \mathrm{~B}}$ e Oliveira, T.G.S. ${ }^{1 \mathrm{C}}$
}

'Universidade Federal de São João del-Rei. São João del Rei. Brasil. *carlabrighenti@ufsj.edu.br; Adeodoro@ufsj.edu.br; Byolaserpa@gmail.com; Coliveira.thelmo@yahoo.com.br

\section{Palavras chave adicionaIS}

Apis mellifera. Baccharis. Emergência de rainhas. Longevidade. Ritmo circadiano.

\section{RESUMO}

A estatística circular é aplicada a dados em que é possível notar um comportamento periódico que caracteriza a obtenção dos chamados dados circulares. A existência de um ritmo circadiano no metabolismo de abelhas africanizadas pode influenciar no horário de mortalidade de operárias e de emergência de rainhas, sendo obtidas nesse caso, medidas circulares, caracterizadas pelo horário de ocorrência de tais eventos. As visitas de abelhas às flores de uma planta podem, igualmente, fornecer dados circulares. A finalidade do trabalho foi verificar aplicabilidade da estatística circular em dados apícolas. Utilizaram-se os dados de três experimentos: 1- forrageamento de abelhas em alecrim do campo; 2- emergência de rainhas e 3- horário de mortalidade de operárias (considerando-se o horário de montagem de experimento à noite (19 h $30 \mathrm{~min}$ ) e durante o dia (7 h $30 \mathrm{~min}$ )). Todos os conjuntos de dados utilizados foram testados em relação a duas distribuições: uniforme e Von Mises (ou normal circular) e foram ajustados à segunda. Concluiu-se que, para o forrageamento de abelhas no alecrim o modelo da normal circular é com um horário médio de forrageamento igual a $11 \mathrm{~h} 29 \mathrm{~min}$ com grande dispersão. Existe também um ritmo circadiano que regula o horário de emergência de rainhas e de mortalidade das abelhas operárias. Para o experimento de emergência de rainhas obteve-se o intervalo de confiança a $95 \%$ para a média circular igual a (4 h 12 min; 9 h 05 min). O horário médio de mortalidade no caso da montagem realizada du-

\section{AdDitiOnAL KEYWORDS}

Apis mellifera. Baccharis. Circadian rhythm. Emergence of queens. Longevity.

rante o dia ocorre após $4 \mathrm{~h} 18 \mathrm{~min}$, com longevidade estimada em 8 h e $37 \mathrm{~min}$, enquanto para indivíduos mantidos sob as mesmas condições, sendo alterado apenas o horário de montagem do experimento para as $19 \mathrm{~h} 30 \mathrm{~min}$, estima-se uma longevidade de 19 horas e 37 minutos. É recomendável, portanto que, para a execução de experimentos laboratoriais com adultos de abelhas, seja incluído na metodologia experimental o horário de montagem do experimento.

\section{SUMMARY}

Circular statistics is applied to data that show a periodic behavior, which characterizes the obtaining of what is called circular data. The existence of a circadian rhythm in the metabolism of Africanized bees can influence the mortality time of workers and the emergence of queen bees, in which case we obtain circular data characterized by the time of occurrence of these events. Bees' visits to flowers of a plant can also provide circular data. The aim of this research was to verify the applicability of circular statistics to apicultural data. Data from three experiments were used: 1 - bees foraging for field rosemary; 2emergence of queen bees; 3 - the mortality time of workers (considering the experimental setup time at night (19:30) and during the day (7:30)). All data sets used were tested in relation to two distributions: uniform and Von Mises (or circular normal), and fitted to the latter. It was concluded 
that, when bees are foraging on rosemary, the circular normal model mean time equals $11 \mathrm{~h} 29 \mathrm{~min}$ with large dispersion. There is also a circadian rhythm that regulates the emergence time of queens and the mortality of worker bees. In the experiment on the emergence of queen bees, a confidence interval of $95 \%$ was obtained for the circular mean equal to ( $4 \mathrm{~h} 12 \mathrm{~min} ; 9 \mathrm{~h} 05 \mathrm{~min}$ ). The mean mortality time in the setup carried out during the day occurs after $4 \mathrm{~h} 18 \mathrm{~min}$, with longevity estimated at $8 \mathrm{~h} 37$ $\min$. The life expectancy of individuals maintained under the same conditions, except for an experimental setup time at $19 \mathrm{~h} 30 \mathrm{~min}$, is estimated at 19 h $37 \mathrm{~min}$. Therefore, in order to carry out laboratory experiments on adult bees, it is advisable to include the experimental setup time in the experimental methodology.

\section{INTRODUÇÃO}

Quando o objetivo do pesquisador é modelar fenômenos que acontecem com frequencia periódica, normalmente, são registrados os chamados dados angulares, direcionais ou circulares. Estes são encontrados em todas as áreas das ciências aplicadas, presumivelmente mais proeminentes em ciências animal e ambiental (Arnold e SenGupta, 2006). Uma observação de uma variável circular pode ser vista como um ponto $\mathrm{P}$ sobre o círculo unitário, centrado na origem $O$ do sistema de coordenadas cartesianas e, portanto, tem-se que $\overrightarrow{O P}$ é um vetor unitário em $\mathrm{R}^{2}$ (Ibrahim et al., 2013), tal como os registrados nas horas do dia, sendo estes transformados em dados direcionais através de variações de ângulos representativos.

Em alguns experimentos é comum estar interessado no horário de ocorrência de um acontecimento, como por exemplo, o horário de forrageamento de insetos a plantas (Malerbo-Souza e Halak, 2009) ou mesmo do parto de equinos os quais normalmente ocorrem com maior incidência a partir das 22 horas (Finger et al., 2010).

A principal característica que diferencia os dados circulares dos dados medidos em uma escala linear é sua natureza, ou seja, o início coincide com o fim em cada período completo, e em geral, com a média $\mu$ sendo a mesma $y+2 p \pi$ para qualquer inteiro $p$ (Otieno, 2002).

Seja $y_{1} \ldots y_{n}$, uma amostra aleatória de tamanho $n$ de uma população circular com função de distribuição $F(y)$. O ângulo $\mathrm{y}_{1}$ representa a unidade de um vetor $O P$ fazendo ângulo $\mathrm{y}_{1}$ com o semi-eixo $O_{x}$ na direção positiva (medido no sentido antihorário), onde $P_{i}$ é o ponto de intersecção no círculo. Assim, uma observação de uma variável circular pode ser representada pelo par ordenado $(\cos (y)$, sen $(y))$, em coordenadas cartesianas, ou pelo par $(1, y)$, em coordenadas polares (Izbicki e Esteves, 2008).

Embora os dados circulares sejam coletados em diferentes tipos de unidades e possam ser expressos em qualquer medida de ângulo, adota-se trabalhar em radianos, com intervalo de variação $[0,2 \pi]$ ou $[-\pi, \pi]$, dependendo do interesse da análise (Batschelet, 1981).

Considerando-se uma amostra aleatória de $n$ observações circulares $\mathrm{y}_{1}, \cdots, \mathrm{y}_{\mathrm{n}}$, às quais se pode associar $n$ vetores unitários $\overline{O P}_{1} \ldots \overline{O P}_{\mathrm{n}}$, calcula-se as seguintes quantidades:

$$
S=\sum_{i=1}^{n} \operatorname{sen}\left(y_{i}\right), \quad C=\sum_{i=1}^{n} \cos \left(y_{i}\right), \quad R^{2}=C^{2}+S^{2}
$$

A média circular de $\mathrm{y}_{1} \ldots \mathrm{y}_{\mathrm{n}}$ é definida como sendo o ângulo $\bar{\mu}$ correspondente ao vetor resultante da soma $\overline{O P}_{1} \ldots \overline{O P}_{\mathrm{n}}$. Esse ângulo deve satisfazer as condições:

$$
\begin{aligned}
& \text { 1) } \cos (\bar{\mu})=C / R \\
& \text { 2) } \operatorname{sen}(\bar{\mu})=S / R \\
& \text { 3) } \bar{\mu}=\left\{\begin{array}{c}
\operatorname{arctg}(S / C), \text { se } S \geq 0 \text { e } C>0, \\
\operatorname{arctg}(S / C)+\pi, \text { se } C<0, \\
\operatorname{arctg}(S / C)+2 \pi, \text { se } S<0 \text { e } C>0 .
\end{array}\right.
\end{aligned}
$$

A quantidade $R=\sqrt{C^{2}+S^{2}}$ representa o comprimento do vetor resultante e fornece 


\section{DISTRIBUIÇÃO VON MISES NA AVALIAÇÃO DE DADOS APÍCOLAS}

informação sobre o grau de concentração dos ângulos observados.

Em vez de $R$, é mais comum usar o comprimento médio do vetor resultante, definido por $\bar{R}=R / n$, o qual tem a vantagem de variar no intervalo $[0,1]$. A variância circular amostral, ou parâmetro de concentração, é definida por $V=1-\bar{R}$, enquanto que o desvio padrão circular é definido por $\sigma=\{-2 \log (1-V)\}^{1 / 2}$, diferentemente da definição usual para dados lineares, onde o desvio padrão corresponde diretamente à raiz quadrada da variância (Izbicki e Esteves, 2008).

A variância mínima ocorre quando $V=0$ $(\bar{R}=1)$, e corresponde a todas as observações estarem precisamente no mesmo local. O maior limite possível de variação ocorre para dados uniformemente distribuídos ao redor do círculo, e corresponde a $V=1(\bar{R}=$ $0)$. O cálculo de $\bar{R}$, portanto, é simples e a interpretação dos resultados não depende de suposições sobre os dados originais (Otieno, 2002).

A distribuição de maior destaque para dados circulares é a chamada Distribuição de von Mises. Ela é tão importante para dados circulares, quanto o modelo Gaussiano é para dados lineares em geral. Esse modelo foi proposto pelo físico alemão R. Von Mises, em 1918, ao investigar desvio de pesos atômicos de valores inteiros (Mardia e Jupp, 2000).

Dizemos que uma variável aleatória circular Y tem distribuição von Mises com parâmetros $\mu$ e $\kappa$ se sua função densidade de probabilidade for dada por:

$$
\begin{aligned}
& f_{Y}(y ; \mu, \kappa)=\frac{1}{2 \pi I_{0}(\kappa)} \exp [\kappa \cos (y-\mu)] \\
& 0 \leq y<2 \pi, \quad 0 \leq \mu<2 \pi \quad \text { e } \quad \kappa>0
\end{aligned}
$$

onde $\mathrm{I}_{0}(\kappa)$ é a função de Bessel modificada do $1^{\circ}$ tipo e ordem zero, que é dada pela série:

$$
I_{0}(\kappa)=\sum_{r=0}^{\infty} \frac{1}{r !^{2}}\left(\frac{1}{2} \kappa\right)^{2 r}
$$

O parâmetro $\mu$ representa a média circular de Y, enquanto $\kappa$ é denominado parâmetro de concentração.

Alguns fenômenos podem ser influenciados tanto por fatores externos quanto pelo ritmo circadiano do próprio animal. Withrow (1959) ${ }^{1}$, citado por Proni e Macieira (2004), introduziu o termo circadiano (latim circa: em torno de; dies: dia), para caracterizar os ritmos com períodos de 24 horas, os quais são sincronizáveis em ciclos de 24 horas de claro/escuro. Segundo Proni e Macieira (2004), a característica de maior interesse no estudo de ritmo de 24 horas, não seria o fato de certas atividades ocorrerem todas neste intervalo, mas sim que tais repetições persistissem na ausência de mudanças ambientais normais. Assim, o ritmo circadiano pode influenciar, principalmente, na ocorrência de eventos de animais com menor ciclo de vida tais como as abelhas.

$\mathrm{O}$ forrageamento de abelhas às flores de uma planta podem fornecer dados circulares já que pode ocorrer sazonalidade tanto na atividade das abelhas em coletar nectar quanto no desabrochamento das flores. O Baccharis dracunculifolia é uma planta arbustiva que ocorre no cerrado brasileiro e popularmente conhecida como alecrim-docampo ou vassourinha (Fagundes et al., 2005). Sabe-se que é através da coleta de resina pelas abelhas africanizadas nesta planta que ocorre a produção da chamada própolis verde, atualmente super valorizada (Maróstica Junior et al., 2008).

Assim é importante no manejo apícola a realização de substituição de rainhas, sendo necessário realizar a produção destas e posterior introdução nas colmeias. A produção de rainhas oferece ao apicultor uma oportunidade singularmente favorável para influenciar o futuro comportamento e

${ }^{1}$ Withrow, R.B. 1959. Photoperiodism and Related Phenomenain Plants and Animals. American Association for the Advancement of Science, 431 pp. 
rendimento produtivo da colônia de abelhas. Por esta razão, há séculos vem sendo desenvolvidos e praticados métodos destinados a produção de rainhas (Silva et al.,1995). Para facilitar o manejo das rainhas para introdução é importante acompanhar o horário de emergência das mesmas, horário no qual estas tem menor movimentação o que auxilia no procedimento de marcação, pesagem e medição.

A existência de um ritmo circadiano no metabolismo desses insetos pode ainda influenciar no horário de mortalidade, sendo este fator importante principalmente para experimentos com abelhas realizados em condições de laboratório.

O objetivo do trabalho foi verificar aplicabilidade da estatística circular em dados oriundos de práticas apícolas, tais como o horário de forrageamento de abelhas ao alecrim do campo, modelagem do horário de emergência das rainhas de abelhas africanizadas, além da verificação da presença do ritmo circadiano na mortalidade de operárias.

\section{MATERIALE MÉTODOS}

As horas do dia foram transformadas em ângulos medidos em radianos de modo que ficassem distribuídos em uma circunferência ( 1 hora $=15^{\circ}=0,2618 \mathrm{rad}$ ) e os horários ao longo das 24 horas foram transformados em vetores de dados circulares. Assim, um dia com duração de 24 horas corresponde a $2 \pi$ rad, ou a aproximadamente $6,28 \mathrm{rad}$.

A implementação computacional das operações para obtenção dos parâmetros da Distribuição de von Mises, foi realizada utilizando-se os pacotes Circular e CircStat do software R Development Core Team. R. (2013). A partir dos dados transformados foram obtidas a média e variancia circular gerou-se o gráfico de dispersão circular e o diagrama do tipo rose diagram no qual obtem-se a distribuição dos dados em função da circunferência.

Realizou-se então o teste de Watson $(\alpha=0,05)$ para verificar o ajustamento dos dados circulares em relação a duas distribuições: uniforme e Von Mises. Quando verificada a adequacidade do ajuste, eram obtidas as estimativas de máxima verossimilhança do parâmetro média circular e $\operatorname{kappa}(\kappa)$, o parâmetro de concentração de uma distribuição von Mises e posteriormente gerada a curva de densidade de probabilidade considerando um intervalo de 48 horas a partir de 0 horas.

\section{HORÁRIO DE FORRAGEAMENTO DE ABELHAS EM ALECRIM DO CAMPO}

As coletas de dados em campo foram realizadas na microrregião de São João Del Rei, na cidade de Tiradentes - Minas Gerais, Brasil, localizada a S $21^{\circ} 07^{\prime}$ e O $44^{\circ} 22^{\prime}$. A vegetação da região é típica de cerrado com áreas muito degradadas. E entre as plantas nativas da região encontra-se o alecrim do campo- Baccharis dracunculifolia. Foram realizadas observações em quatro plantas de alecrim do campo em uma área de $10 \mathrm{~m}^{2}$ próxima a apiário em produção com 12 colônias, durante quatro dias no mês de fevereiro de 2011, período em que ocorre o pico de produtividade de própolis verde na região do estudo. As coletas foram realizadas diariamente das 10 até as 16 horas, registrando-se a hora e minuto da visitação de cada abelha africanizada a cada uma das plantas. Contou-se apenas as abelhas que pousaram nas gemas apicais e coletaram pequenos fragmentos resinosos da planta. Foram registrados também os dados meteorológicos dos dias de coleta obtidos pela estação meteorológica automatizada instalada no CTAN (Campus Tancredo Neves) da Universidade Federal de São João del Rei, em convenio com o Instituto Nacional de Meteorologia (INMET).

\section{HORÁRIO DE EMERGÊNCIA DE RAINHAS}

Para obtenção da colônia matriz foi utilizado o apiário de produção apícola da empresa Linha de Voo localizado no município de Tiradentes-MG $\left(21^{\circ} 07^{\prime} \mathrm{S}, 44^{\circ} 22^{\prime}\right.$ $\mathrm{W}$ e $923 \mathrm{~m}$ de altitude). Entre as colmeias 


\section{DISTRIBUIÇÃO VON MISES NA AVALIAÇÃO DE DADOS APÍCOLAS}

pertencentes ao apiário, foi selecionada, a partir de critérios de produtividade de própolis e de comportamento higiênico a colonia para fornecimento de ovos para produção das rainhas.

O método de produção de rainhas utilizado foi o Nicot, ou também denominado de cupularvae. Neste método, inicialmente, se insere um quadro de acrílico para oviposição da rainha matriz. Este quadro possui 110 cúpulas das quais se obtém, após 3 dias, ovos que serão transferidos para quadro porta-cúpulas e introduzidos na colmeia recria até formação da realeira. Após um período de 10 dias na colmeia recria, as realeiras assim induzidas, foram transportadas e mantidas em câmara climatizada a $29 \pm$ $2{ }^{\circ} \mathrm{C}$, UR $70 \pm 10 \%$ e sob ausência de luz. A partir deste momento, as realeiras foram avaliadas a cada hora, registrando-se a emergência das rainhas.

\section{HORÁRIO DE MORTALIDADE DE OPERÁRIAS}

A estatística circular foi aplicada a dados obtidos de dois experimentos realizados com o objetivo de estudar o horário de mortalidade de abelhas ao longo do dia e verificar a interferência do horário de montagem de experimentos em laboratório na longevidade de abelhas. As unidades experimentais foram gaiolas de PVC de 10 $\mathrm{cm}$ de diâmetro por $5 \mathrm{~cm}$ de altura, tendo a parte superior revestida com tecido tipo filó e a inferior com organza. Em cada unidade experimental foram colocados dez adultos com até 1 hora de idade, obtidos de uma núcleo de emergência denominada Emergapis.

O núcleo tem $50 \mathrm{~cm}$ de comprimento $\mathrm{x} 3,5$ $\mathrm{cm}$ de largura x $25 \mathrm{~cm}$ de profundidade, permitindo a colocação de um quadro de ninho. Sua estrutura é de madeira revestida de tela plástica de malha de $2 \mathrm{~mm}$, cor branca, possibilitando visualizar a emergência dos insetos no seu interior. A parte superior é confeccionada em madeira podendo ser removida para inserção do quadro. A parte inferior tem estrutura semelhante e móvel permitindo sua remoção para eliminar adul- tos mortos. O núcleo foi mantido em câmara climatizada a $34 \pm 2{ }^{\circ} \mathrm{C}$, UR de $70 \pm 10 \%$ e ausência de luz. Assim, pode-se adquirir os indivíduos com idade conhecida para avaliar a parte experimental propriamente dita.

As abelhas nas gaiolas foram então mantidas em câmara climatizada a $29 \pm 2{ }^{\circ} \mathrm{C}$, UR $70 \pm 10 \%$ e fotoperíodo de 12 horas. Em cada experimento foram dispostas 10 gaiolas com água apenas ad libitum e avaliada a mortalidade ao longo do tempo. A diferença básica entre os dois experimentos é o horário de introdução das abelhas nas gaiolas, sendo que um dos experimentos foi montado no início da manhã as 7 h e 30 min e o outro durante a noite as $19 \mathrm{~h}$ e $30 \mathrm{~min}$. O horário de mortalidade de cada grupo de 100 abelhas africanizadas foi registrado ao longo de 24 horas.

\section{RESULTADOSEDISCUSSÃO}

Todos os conjuntos de dados foram testados em relação a duas distribuições: uniforme e Von Mises. Eles foram ajustados à distribuição Von Mises (p>0,05). Para cada experimento foram construídos os diagramas de dispersão e estimados os parâmetros para plotagem da curva da distribuição Von Mises correspondente.

\section{HORÁRIODE FORRAGEAMENTODE ABELHAS EM ALECRIM DOCAMPO}

Os dados meteorológicos médios obtidos nos horários e dias de coletas foram: Temperatura oscilando entre 22,9 e $24,8{ }^{\circ} \mathrm{C}$, Umidade entre 62 e $71 \%$, Radiação média de $1682 \mathrm{~kJ} / \mathrm{m}^{2}$ e vento com velocidade média de $2,86 \mathrm{~m} / \mathrm{s}$. Notou-se também que nos dias de coleta avaliados, houve dois dias ensolarados e dois nublados, no entanto, não houve diferença estatisticamente significativa no ajuste de forrageamento entre estas duas características $(\mathrm{p}>0,05)$ e portanto, optou-se por analisar os dados em conjunto.

Foram observadas 208 abelhas africanizadas no alecrimcom média de $13 \pm 2,69$ abelhas/planta/dia. Notou-se também a 


\section{BRIGHENTI, BRIGHENTI, MIRANDA E OLIVEIRA}

ocorrência de outras espécies de abelhas, não para a coleta de resina, tais como Melipona quadrifasciata e Tetragonisca angustula, mas que não foram registradas por não se tratar do objetivo principal deste trabalho. A partir dos horários registrados de forrageamento de abelhas em cada planta de alecrim do campo, foi possível construir um diagrama de rosas . No gráfico de dispersão circular o sentido adotado é o anti-horário e para estes dados, como houve restrição do horário de acompanhamento de visitação, o ciclo completo corresponde a apenas 6 horas (entre 10 e 16 horas). Assim, cada $90^{\circ}$ (ou $\pi / 2 \mathrm{rad}$ ) correspondente a 1 hora e 30 minutos, ou seja, $180^{\circ}$ ou $\pi$ equivale a uma hora da tarde e assim sucessivamente (figura 1).

Pode-se notar uma dispersão da visitação durante todo o intervalo de tempo avaliado. Há um menor forrageamento entre 13 h e 14 h 30 min. Através da observação do horário registrado de visitação por cada abelha, pode-se ajustar um modelo de distribuição circular de Von Mises para o horário de forrageamento das abelhas no alecrim do campo. Para tal ajuste, determinaram-se ini-
Tabela I. Parâmetros circulares ajustados aos dados de modelagem do forrageamento de abelhas africanizadas em alecrim do campo. (Circular parameters fitted to the modelling data of Africanized bees foraging in the field rosemary).

\begin{tabular}{lc}
\hline № de visitas observadas & 208 \\
Parâmetro de concentração $K$ (rad) & 0,172 \\
Média circular $\bar{\mu}$ (rad) & 1,563 \\
$\bar{\mu}$ (horas) & $11 \mathrm{~h} 29 \mathrm{~min}$ \\
\hline
\end{tabular}

cialmente os parâmetros de media circular e de dispersão (tabela I).

A média direcional igual a 1,563 radianos corresponde a $89,5^{\circ}$, ou seja, aproximadamente às 11 h 29 min (tabela I). Com os parâmetros estimados foi possível plotar a função distribuição de probabilidade correspondente (figura 2).

Percebe-se que há um aumento no fluxo de forrageamento com ápice em 1,56 radianos (às 11 h 29 min) e a partir daí um decréscimo na visitação das abelhas ao alecrim do campo, estimando-se a menor incidência do forrageamento em 4,71 rad (às 14 h $30 \mathrm{~min}$ ) ocorrendo a partir daí um novo

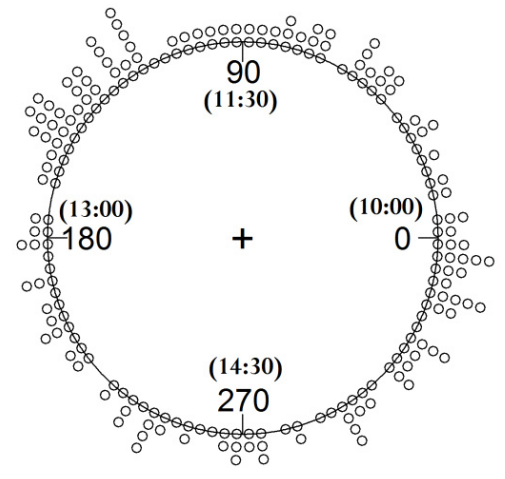

A

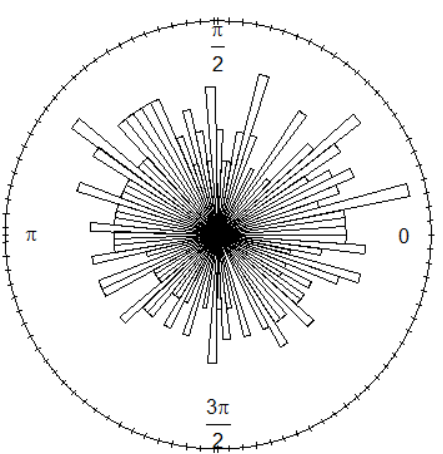

B

Figura 1. Representação angular das observações do horário de forrageamento de abelhas africanizadas em alecrim do campo. (A) Gráfico de dispersão e (B) Diagrama de rosas. (Angular representation of observations of forage time of Africanized bees in the field rosemary. (A) Scatter plot and (B) Rose diagram).

Archivos de zootecnia vol. 63, núm. 243, p. 466. 


\section{DISTRIBUIÇÃO VON MISES NA AVALIAÇÃO DE DADOS APÍCOLAS}

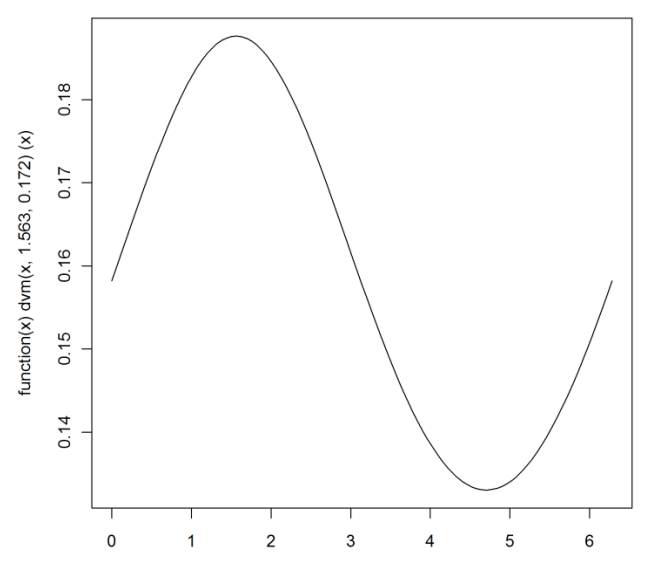

Figura 2. Densidade de probabilidade para observação da visitação de abelhas africanizadas em alecrim do campo ajustados a distribuição de Von Mises, considerando o intervalo de 6 horas a partir das 10 horas $(0$ radianos) até as 16 horas $(2 \pi \mathrm{rad})$. (Probability density for the observation of Africanized bees' visits to field rosemary fitted to Von Mises distribution, considering the 6-hour interval from 10 hours ( 0 radians) to 16 hours ( $2 \pi$ radians)).

aumento de fluxo (figura 2). Portanto, não se recomenda o manejo das colmeias no intervalo de $12 \mathrm{~h}$ as $14 \mathrm{~h}$, pois neste período há um decréscimo no forrageamento e portanto um número maior de indivíduos na colônia.

Com os dados de radiação nos quatro dias de registro de forrageamento, avaliouse que o horário de menor fluxo de forrageamento é também aquele em que ocorre aumento no índice de radiação, o qual decresce a partir das 15 horas (figura 3). Este fator pode ainda influenciar o fluxo de forrageamento já que este fator, está diretamente relacionado a disponibilidade de compostos voláteis que podem alterar a atratividade dos insetos no forrageamento.

\section{HORÁRIO DE EMERGÊNCIA DE RAINHAS}

Durante o período do experimento houve emergência de 42 rainhas e os horários foram transformados em dados circulares, ou seja, transformou os horários em radianos, de modo que cada hora representa-se 0,26 rad ou 15 graus.

A partir do horário de emergência de cada rainha, foi construído o gráfico de dispersão circular, no qual o sentido adotado é o anti-horário, sendo $90^{\circ}$ correspondente a 6 horas da manhã e $180^{\circ}$ equivale ao meio dia e assim respectivamente. No diagrama de rosas evidencia-se também a representação do horário de emergência das rainhas, neste caso obtendo-se o horário em radianos (figura 4).

Nota-se que há maior concentração de emergência entre o fim do primeiro quadrante e início do segundo, o que equivale a aproximadamente 3 horas e 8 horas, ocorrendo o menor índice de emergnecia no quarto quadrante que representa o período entre 18 e 24 horas.

As medidas circulares de dispersão foram obtidas segundo o ajuste à distribuição e estão representadas na tabela II.

Obteve-se o intervalo de confiança a $95 \%$ para a média circular, sendo igual a $[1,1 ; 2,38]$ em radianos, o que equivale a [ $4 \mathrm{~h}$ 12 min; 9 h 5 min] com parâmetro de concentração igual a 0,777 que equivale a 3 horas . A curva normal circular ajustada a partir destes parâmetros está representada na figura 5.

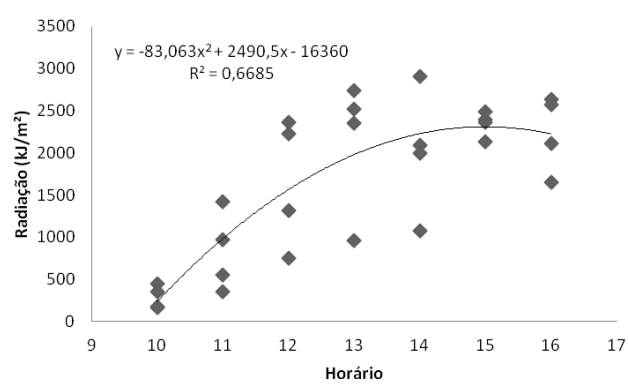

Figura 3. Valores de radiação em função do horário nos dias de observação do forrageamento de abelhas africanizadas em alecrim do campo. (Radiation values according to hours on the days of observation of Africanized bees foraging in the field rosemary). 
A

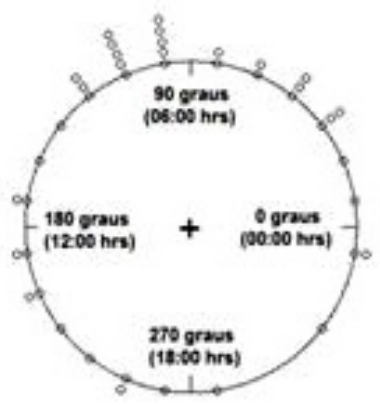

B

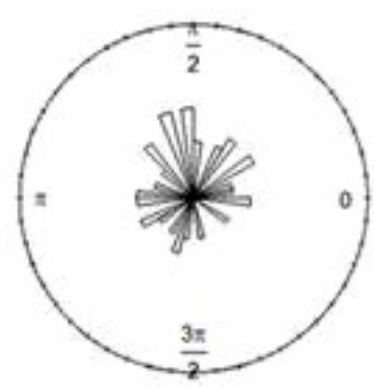

Figura 4. Representação angular das observações do horário de emergência de rainhas de abelhas africanizadas. (A) Gráfico de dispersão e (B) Diagrama de rosas. (Angular representation of the observed times of emergence of Africanized queen bees. (A) Scatter plot and (B) Rose diagram).

Nota-se um aumento crescente na emergência a partir das 0 horas $(0 \mathrm{rad})$ com um máximo as $1,848 \mathrm{rad}(7 \mathrm{~h} 03 \mathrm{~min})$ a partir do qual ocorre o decréscimo até 4,99 rad (19 h 03 min), evidenciando que a emergência de rainhas é reduzida no período diurno, ocorrendo com maior intensidade no período noturno.

\section{HORÁRIO DE MORTALIDADE DE OPERÁRIAS}

Os indivíduos de abelhas africanizadas recém-emergidos coletados do Emergapis para montagem do experimento possuiam pouca atividade motora e por isso não se fez necessária a utilização de $\mathrm{CO}_{2}$ para anes-

Tabela II. Parâmetros circulares para modelagem do horário de emergência de rainhas de abelhas africanizadas. (Circular parameters for modelling the hour of emergence of Africanized queen bees).

\begin{tabular}{lc}
\hline № de rainhas recém-emergidas & 42 \\
$\bar{R}$ & 0,362 \\
$\nu$ & 0,638 \\
$\kappa$ & 0,777 \\
$\bar{\mu}$ (rad) & 1,848 \\
$\bar{\mu}$ (horas) & $07 \mathrm{~h} \mathrm{03}$ min
\end{tabular}

$\bar{R}=$ comprimento médio resultante; $v=$ variância circular; $\kappa=$ parâmetro de concentração; $\bar{\mu}(\mathrm{rad})=$ média circular (rad); $\bar{\mu}(\mathrm{h})=$ média circular $(\mathrm{h})$. tesiá-los como é de rotina em experimentos realizados com A. mellifera. Podendo desta maneira, prolongar a sobrevida desses insetos, já que, segundo Czekonska (2007), a exposição ao dióxido de carbono afeta seu desenvolvimento. Outra vantagem da utilização do emergapis é a independência de fatores climáticos para a montagem dos experimentos, já que não é necessário estar no campo para coleta das abelhas e a precisão da idade dos indivíduos para cálculos de

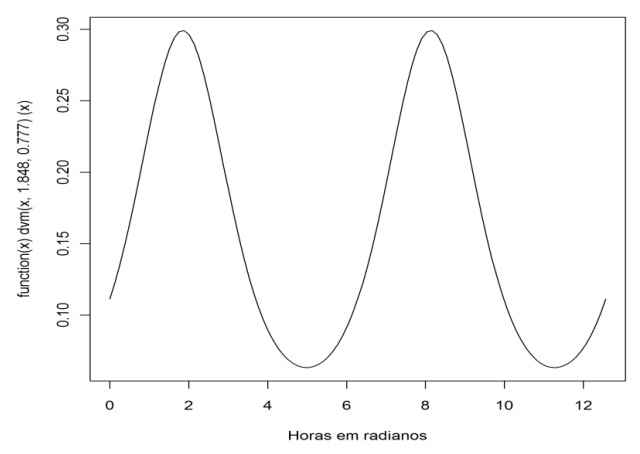

Figura 5. Densidade de probabilidade para observação de emergência de rainhas de abelhas africanizadas ajustados a distribuição de Von Mises $H_{E R} \sim V M(1,848 ; 0,777)$, considerando o intervalo de 48 horas a partir das 0 horas. (Probability density for observing the emergence of Africanized queen bees fitted to the Von Mises distribution HER VM (1.848;0.777), considering the 48 -hour interval from 0 hours).

Archivos de zootecnia vol. 63, núm. 243, p. 468. 


\section{DISTRIBUIÇÃO VON MISES NA AVALIAÇÃO DE DADOS APÍCOLAS}

Tabela III. Número de operárias de abelhas Africanizadas mortas em função do tempo decorrido do horário de montagem do experimento. (Number of dead Africanized worker bees according to time elapsed since experimental setup).

\begin{tabular}{lcccccccc}
\hline Horário de & Intervalo de tempo decorrido após a montagem (h) & \multicolumn{2}{c}{ Total de } & Tempo médio \\
montagem & $0-4$ & $4-8$ & $8-12$ & $12-16$ & $16-20$ & $20-24$ & abelhas mortas & de vida (h) \\
\hline Dia- 7:30 & 2 & 68 & 25 & 0 & 3 & 1 & 99 & 7,69 \\
Noite- 19:30 & 0 & 0 & 2 & 15 & 76 & 7 & 100 & 21,37 \\
\hline
\end{tabular}

longevidade. É importante ressaltar que a capacidade de oviposição de uma rainha africanizada pode chegar a 4000 mil ovos por dia, assim, foi possível adquirir os 200 indivíduos para montagem do experimento num intervalo inferior a 60 minutos, tanto no período da manhã quanto na montagem da noite.

O número total de indivíduos mortos a cada 4 horas decorridos do experimento foi registrado e os resultados apresentados na tabela III.

Percebe-se que a maior ocorrência de mortalidade de abelhas no experimento montado durante o dia ocorreu entre 4 e 8 horas após a montagem do mesmo, ou seja, entre $11 \mathrm{~h} 30 \mathrm{~min}$ e $15 \mathrm{~h} 30 \mathrm{~min}$. É interessante ressaltar que no experimento montado a noite, o pico de mortalidade ocorreu após 16 e 20 horas da montagem, o que corresponde também ao intervalo vespertino do experimento diurno, indicando que, realmente, há um prolongamento de aproximadamente 12 horas na longevidade desses insetos.

Com os dados de horário de mortalidade das abelhas nos experimentos montados com 12 horas de diferença entre eles, foram construídos os gráficos de dispersão circular (figura 6).

Observa-se que as mortes das abelhas se concentram no segundo quadrante na montagem realizada durante o dia e no quarto quadrante na montagem realizada durante a noite. Assim, evidencia-se uma diferença de $\pi \mathrm{rad}$ ou $180^{\circ}$, o que equivale a 12 horas, indicando que a montagem realizada no período noturno prolongou a longevidade das abelhas operárias, que sobreviveram 12 horas a mais do que aquelas que foram submetidas ao mesmo ensaio no período da manhã.

As medidas circulares dos dados ajustados foram então obtidas e apresentadas na tabela IV.

No caso da montagem realizada durante o dia, o horário médio de mortalidade ocorre após 4 h 18 min da montagem do experimento, então, estima-se, pela ajuste da distribuição Von Mises, uma longevidade de $8 \mathrm{~h}$ e 37 min para os indivíduos adultos mantidos em experimentos montados pela manhã. Enquanto que, para indivíduos mantidos sob as mesmas condições, sendo alterado apenas o horário de montagem do experimento para as 19 h 30 min, estima-se uma

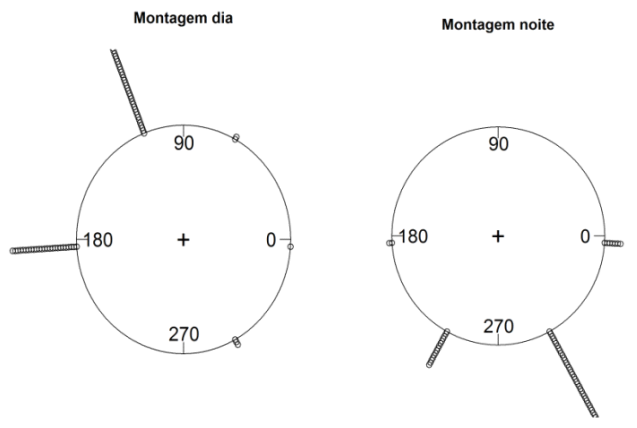

Figura 6. Gráfico circular de dispersão das observações do horário de mortalidade de operárias de abelhas Africanizadas em experimentos montados durante o dia e à noite. (Circular scatter plot for the observations of mortality time of Africanized worker bees in experiments set up during the day and the evening). 
Tabela IV. Parâmetros circulares ajustados aos dados de horários de mortalidade de operárias de abelhas Africanizadas em experimentos montados durante o dia e à noite. (Circular parameters adjusted to the data on the mortality time of Africanized worker bees in experiments set up during the day and the evening).

\begin{tabular}{lcc}
\hline Horário de montagem & Dia & Noite \\
\hline № abelhas mortas & 96 & 82 \\
$\bar{R}$ & 0,795 & 0,864 \\
$\nu$ & 0,205 & 0,136 \\
$\kappa$ & 2,804 & 3,994 \\
$\bar{\mu}$ (rad) & 2,256 & 5,133 \\
$\bar{\mu}$ (h) & $08 \mathrm{~h} 37$ min & $19 \mathrm{~h} 37$ min
\end{tabular}

$\bar{R}=$ comprimento médio resultante; $v=$ variância circular; $\kappa=$ parâmetro de concentração; $\bar{\mu}(\mathrm{rad})=$ média circular (rad); $\bar{\mu}(\mathrm{h})=$ média circular $(\mathrm{h})$.

longevidade de 19 horas e 37 minutos, ou seja 11 horas a mais de sobrevivência influenciada apenas por este aspecto. Notase um atraso na mortalidade das abelhas quando a montagem do experimento é realizada durante o período noturno. Segundo Southwick (1992), citado por Brighenti et al. (2008), um inseto exposto a menor quantidade de luz tem menor atividade e, consequentemente, um possível aumento de sua longevidade. Portanto, é importante considerar o horário de montagem do experimentos quando estes são de curta duração pois a diferença de horário ao se avaliar, por exemplo, a mortalidade pode ser sub ou superestimada em relação aos valores do tempo de atuação de um inseticida, por exemplo.

De acordo com a curva normal circular, os picos na figura 7 representam a média de tempo até ocorrência da mortalidade. $\mathrm{Na}$ montagem à noite, os picos são mais altos e estreitos, o que demonstra a concentração das mortes em determinado horário. Durante a montagem de dia, as mortes são um pouco mais dispersas, o que é confirmado pelos picos mais baixos e largos.

\section{CONCLUSÕES}

A estatística circular se mostrou adequada pra avaliação de dados envolvendo ritmo circadiano em todas os ensaios apícolas realizados.

Obteve-se o pico de forragemaento das abelhas africanizadas no alecrim do campo e verificou-se o ritmo circadiano no horário de emergência de rainhas dessa mesma espécie.

Notou-se a existência de um ritmo circadiano que regula o horário de mortalidade das abelhas operárias de abelhas africanizadas confinadas.

É recomendável, portanto, que, para a execução de experimentos laboratoriais com adultos de abelhas, seja incluída na metodologia experimental o horário de montagem do experimento.

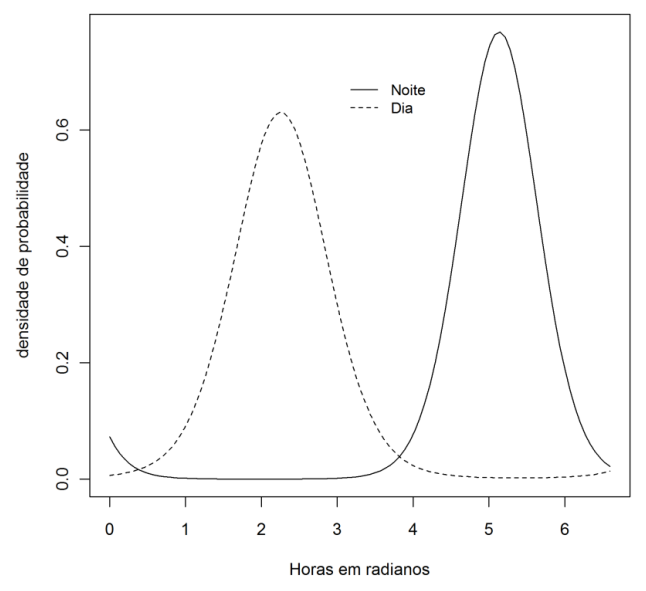

Figura 7. Densidade de probabilidade para observação do horário de mortalidade de operárias de abelhas Africanizadas em experimentos montados durante o dia e à noite ajustados a distribuição de Von Mises, considerando o intervalo de 24 horas $(2 \pi \mathrm{rad})$ a partir das 0 horas. (Probability density for the observation of the Africanized worker bees' mortality time in experiments set up during the day and the evening adjusted to the Von Mises distribution considering the 24-hour interval $(2 \pi \mathrm{rad})$ from 0 hours). 


\section{DISTRIBUIÇÃO VON MISES NA AVALIAÇÃO DEDADOS APÍCOLAS}

\section{AGRADECIMENTOS \\ À FAPEMIG e CNPq pela bolsa de}

\section{BIBLIOGRAFIA}

Arnold, B.C. and SenGupta, A. 2006. Recent advances in the analyses of directional data in ecological and environmental sciences. Environ Ecol Stat, 13: 253-256.

Batschelet, E. 1981. Circular statistics in biology. Academic Press. New York.

Brighenti, C.R.G.; Cirillo, M.A. e Brighenti, D.M. 2008. Análise longitudinal na determinação do fotoperíodo adequado para criação de abelhas em laboratório. Rev Bras Biom, 26: 111-124.

Czekonska, K. 2007. Influence of carbon dioxide on Nosema apis infection of honeybees (Apis mellifera). J Invertebr Pathol, 95: 84-86.

Fagundes, M.; Neves, F.S. and Fernandes, G.W. 2005. Direct and indirect interactions involving ants, insect herbivores, parasitoids, and the host plant Baccharis dracunculifolia (Asteraceae). Ecol Entomol, 30: 28-35.

Finger, I.S.; Curcio, B.R.; Lins, L.A. e Frey, Jr.F. 2010. Assistência ao parto em equinos. Braz J Equine Med, 5: 32-35.

Izbicki, R. e Esteves, L.G. 2008. Análise de dados circulares. IV Simpósio de Iniciação Científica e Pós-Graduação do IME-USP, São Paulo. Atas. pp. 69-74.

Ibrahim, S.; Rambli, A.; Hussin, A.G. and Mohamed, I. 2013. Outlier detection in a circular regression model using COVRATIO Statistic. Commun Stat Simulat C, 42: 2272-2280.

Malerbo-Souza, D.T. e Halak, A.L. 2009. Comportamento de forrageamento de abelhas e outros insetos nas panículas da mangueira
Iniciação Científica concedida ao terceiro e quarto autores.

(Mangifera indica L.) e produção de frutos. Acta Scientiarum. Anim Sci, 31: 335-341.

Mardia, K.V. and Jupp, P.E. 2000. Directional Statistics. John Wiley \& Sons LTD. London.

Maróstica Junior, M.R.; Daugsch, A.; Moraes, C.S.; Queiroga, C.L.; Pastore, G.M. and Park, Y.K. 2008. Comparison of volatile and polyphenolic compounds in Brazilian green propolis and its botanical origin Baccharis dracunculifolia. Cienc Tecnol Aliment, 28: 178181.

Otieno, B.S. 2002. An alternative estimate of preferred direction for circular data. Tese (Doutorado de Filosofia em Estatística). Virginia Polytechnic Institute and State University. Blacksburg, Virginia. $219 \mathrm{f}$.

Proni, E.A. e Macieira, O.J. D. 2004. Ritmo circadiano da taxa respiratória de Tetragonisca angustula fiebrigi (Schwarz), T. angustula (Latreille) e Trigona spinipes (Fabricius) (Hymenoptera, Apidae, Meliponinae). Rev Bras Zool, 21: 987993.

R Development Core Team. R. 2013. A language and environment for statistical computing. $R$ Foundation for Statistical Computing. Vienna, Austria. http://www.R-project.org (10/09/ 2013).

Silva, E.C.A.; Silva, R.M.B.; Chaud-Netto, J.; Moreti, A.C.C.C. and Otsuk, I.P. 1995. Influence of management and environmental factors on mating sucess of Africanized queen honey bees. J Apic Res, 4: 169-175. 